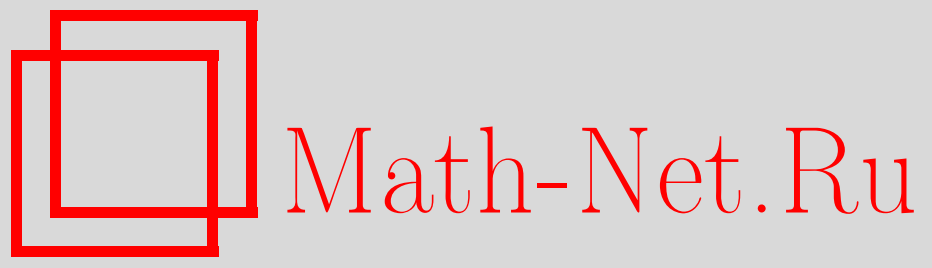

Y. F. Wu, Some limit theorems for arrays of rowwise pairwise NQD random variables, Теория вероятн. и ее примен., 2014, том 59, выпуск 2, 400-410

DOI: https://doi.org/10.4213/tvp4573

Использование Общероссийского математического портала Math-Net.Ru подразумевает, что вы прочитали и согласны с пользовательским соглашением http://www . mathnet.ru/rus/agreement

Параметры загрузки:

IP: 35.174 .16 .151

26 апреля 2023 г., $17: 08: 49$

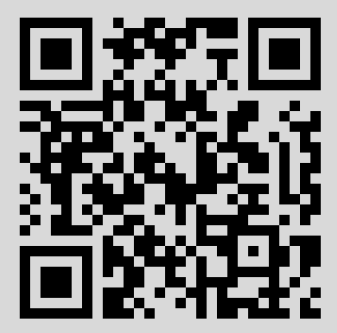


(c) 2014 г.

WU Y.F.*

\section{SOME LIMIT THEOREMS FOR ARRAYS OF ROWWISE PAIRWISE NQD RANDOM VARIABLES ${ }^{1)}$}

В статье изучаются некоторые предельные теоремы для серий случайных величин, в каждой серии обладающих свойством попарно отрицательной зависимости в квадрантах. Решается открытая проблема, поставленная в работе [1]. При соответствующих условиях изучается сходимость в среднем в степени $p$ и полная сходимость.

Ключевые слова и фразы: сходимость в среднем; полная сходимость; равномерная интегрируемость; случайные величины, попарно отрицательно зависимые в квадрантах.

1. Introduction. The concept of negatively quadrant dependent (NQD) random variables was introduced by Lehmann [2].

D e f i n it i o n 1.1. Two random variables $X$ and $Y$ are said to be negatively quadrant dependent (NQD) if

$$
\mathbf{P}(X \leqslant x, Y \leqslant y) \leqslant \mathbf{P}(X \leqslant x) \mathbf{P}(Y \leqslant y) \text { for all } x \text { and } y .
$$

A sequence of random variables $\left\{X_{n}, n \geqslant 1\right\}$ is said to be pairwise NQD if every pair of random variables in the sequence are NQD.

The concept of negatively associated (NA) random variables was introduced by JoagDev and Proschan [3].

$\mathrm{D}$ e $\mathrm{f}$ i $\mathrm{n}$ i t i o $\mathrm{n}$ 1.2. A finite family of random variables $\left\{X_{k}, 1 \leqslant k \leqslant n\right\}$ is said to be negatively associated (abbreviated to NA) if for any disjoint subsets $A$ and $B$ of $\{1,2, \ldots, n\}$ and any real coordinate-wise nondecreasing functions $f$ on $\mathbf{R}^{A}$ and $g$ on $\mathbf{R}^{B}$,

$$
\operatorname{Cov}\left(f\left(X_{i}, i \in A\right), g\left(X_{j}, j \in B\right)\right) \leqslant 0
$$

whenever the covariance exists. An infinite family of random variables is NA if every finite subfamily is NA.

The concept of linearly negative quadrant dependent (LNQD) random variables was introduced by Newman [4].

$\mathrm{D}$ e f i n i t i o n 1.3. A sequence $\left\{X_{n}, n \geqslant 1\right\}$ of random variables is said to be LNQD if for all finite disjoint subsets $A, B \subset \mathbf{N}$ and $r_{j}, r_{k}>0$, the random variables $\sum_{j \in A} r_{j} X_{j}$ and $\sum_{k \in B} r_{k} X_{k}$ are NQD.

As we know, sequences of pairwise NQD random variables are a family of very wide scope, which contains sequences of NA random variable and LNQD random variables. The convergence properties of pairwise NQD random sequences have been studied in many papers. We refer to $\mathrm{Wu}[5]$ for the moment inequalities, [6]-[8] for the strong convergence, [9], [10] for the weak convergence, [5], [11], [12] for the complete convergence, [13], [1], [14] for the mean convergence.

In what follows, let $\left\{u_{n}, n \geqslant 1\right\}$ and $\left\{v_{n}, n \geqslant 1\right\}$ be two sequences of integers (not necessarily positive or finite) such that $v_{n}>u_{n}$ for all $n \geqslant 1$ and $l_{n}:=v_{n}-u_{n} \rightarrow \infty$ as $n \rightarrow \infty$. Let $\left\{k_{n}, n \geqslant 1\right\}$ be a sequence of positive numbers such that $k_{n} \rightarrow \infty$ as $n \rightarrow \infty$.

Sung, Lisawadi, and Volodin [1] introduced the following concept of integrability.

* College of Mathematics and Computer Science, Tongling University, Tongling 244000, China; e-mail: wyfwyf@126.com

1) This work was supported by the Humanities and Social Sciences Foundation for the Youth Scholars of Ministry of Education of China (12YJCZH217), the Natural Science Foundation of Anhui Province (1308085MA03) and the Anhui Province College Excellent Young Talents Fund Project of China (2011SQRL143). 
$\mathrm{D}$ e f i $\mathrm{n}$ i t i o $\mathrm{n}$ 1.4. Let $\left\{X_{n i}, 0 \leqslant i \leqslant l_{n}, n \geqslant 1\right\}$ be an array of random variables and $r>0$. Let moreover $\{h(n), n \geqslant 1\}$ be an increasing sequence of positive constants with $h(n) \uparrow \infty$ as $n \uparrow \infty$. The array $\left\{X_{n i}\right\}$ is said to be $h$-integrable with exponent $r$ if

$$
\sup _{n \geqslant 1} k_{n}^{-1} \sum_{i=0}^{l_{n}} \mathbf{E}\left|X_{n i}\right|^{r}<\infty \quad \text { and } \quad \lim _{n \rightarrow \infty} k_{n}^{-1} \sum_{i=0}^{l_{n}} \mathbf{E}\left|X_{n i}\right|^{r} I\left(\left|X_{n i}\right|^{r}>h(n)\right)=0 .
$$

They studied weak laws of large numbers for the array of dependent random variables satisfying the conditions of $h$-integrability with exponent $r$, and obtained the following theorem.

Theorem A. Suppose that $\left\{X_{n i}, 0 \leqslant i \leqslant l_{n}, n \geqslant 1\right\}$ is an array of rowwise $N A$ integrable with exponent $1 \leqslant r<2$ random variables, $k_{n} \rightarrow \infty, h(n) \uparrow \infty$, and $h(n) / k_{n} \rightarrow$ 0. Then

$$
k_{n}^{-1 / r} \sum_{i=0}^{l_{n}}\left(X_{n i}-\mathbf{E} X_{n i}\right) \rightarrow 0
$$

in $L^{r}$ and, hence, in probability as $n \rightarrow \infty$.

$\mathrm{R}$ e $\mathrm{m}$ a $\mathrm{rk}$ 1.1. Sung, Lisawadi, and Volodin [1] put forward an open problem, that is, whether Theorem A remains true by considering pairwise NQD instead of NA. Wu and Guan [14] extended Theorem A by considering LNQD instead of NA. However, since LNQD implies pairwise NQD, the open problem of [1] is not solved completely in [14].

In this work, we solve this open problem. In addition, we shall study $p$-mean convergence $(p<r)$ and complete convergence for the array of pairwise NQD random variables under some appropriate conditions, which were not considered in [1] and [14].

In this paper, $C$ will denote generic positive constants, whose value may vary from one application to another, $I(A)$ will indicate the indicator function of $A$.

$\mathrm{D}$ e f i n i t i o n 1.5. A sequence of random variables $\left\{X_{n}, n \geqslant 1\right\}$ satisfies the Marcinkiewicz-Zygmund inequality with exponent 2 if

$$
\mathbf{E}\left(\sum_{k=1}^{n} X_{k}\right)^{2} \leqslant C \sum_{k=1}^{n} \mathbf{E} X_{k}^{2}, \quad \text { for all } n \geqslant 1 .
$$

$\mathrm{R}$ e $\mathrm{m}$ a $\mathrm{r} \mathrm{k}$ 1.2. It is worth pointing out that our main methods differ from those used in [1]. The proofs in this paper are based on Definition 1.5 which was established in [15]. According to our knowledge, the following sequences of mean zero random variables satisfy (1.1) with the indicated value of $C$ : independent identically distributed, martingale difference $(C=1,[16])$, pairwise NQD $(C=1,[3]), \varphi$-mixing random variables with $\sum_{n=1}^{\infty} \varphi^{1 / 2}(n)<\infty\left(C=1+4 \sum_{n=1}^{\infty} \varphi^{1 / 2}(n),[17]\right), \rho$-mixing random variables with $\sum_{n=1}^{\infty} \rho(n)<\infty\left(C=1+4 \sum_{n=1}^{\infty} \rho(n)\right.$, [17]). Therefore, following the methods of this paper, we can easily get similar results for the above sequences.

2. Main results. Now we state our main results. The proofs will be given in Section 3.

Theorem 2.1. Let $\left\{X_{n i}, 0 \leqslant i \leqslant l_{n}, n \geqslant 1\right\}$ be an array of rowwise pairwise NQD $h$ integrable with exponent $1 \leqslant r<2$ random variables, $k_{n} \rightarrow \infty, h(n) \uparrow \infty$, and $h(n) / k_{n} \rightarrow$ 0. Then

$$
k_{n}^{-1 / r} \sum_{i=0}^{l_{n}}\left(X_{n i}-\mathbf{E} X_{n i}\right) \rightarrow 0
$$

in $L^{r}$ and, hence, in probability as $n \rightarrow \infty$.

$\mathrm{R}$ e $\mathrm{m}$ a $\mathrm{r} \mathrm{k}$ 2.1. The above theorem shows that if we replace NA by pairwise NQD, Theorem A holds. Therefore, we solve completely the open problem in [1]. 
The following theorem shows that, under the conditions

$$
\begin{gathered}
\sup _{n \geqslant 1} k_{n}^{-1} \sum_{i=0}^{l_{n}} \mathbf{E}\left|X_{n i}\right|^{r}<\infty, \\
\lim _{n \rightarrow \infty} k_{n}^{-1} \sum_{i=0}^{l_{n}} \sup _{y \geqslant h(n)} y \mathbf{P}\left(\left|X_{n i}\right|^{r}>y\right)=0,
\end{gathered}
$$

we can obtain $p$-mean convergence for the array of rowwise pairwise NQD random variables.

Theorem 2.2. Let $\left\{X_{n i}, 0 \leqslant i \leqslant l_{n}, n \geqslant 1\right\}$ be an array of rowwise pairwise NQD random variables and $1<r<2$. Let $k_{n} \rightarrow \infty, h(n) \uparrow \infty$, and $h(n) / k_{n} \rightarrow 0$. Suppose (2.1) and (2.2) hold, then for all $p \in(0, r)$

$$
k_{n}^{-1 / r} \sum_{i=0}^{l_{n}}\left(X_{n i}-\mathbf{E} X_{n i}\right) \rightarrow 0
$$

in $L^{p}$ and, hence, in probability as $n \rightarrow \infty$.

Finally, we state complete convergence for the array of rowwise pairwise NQD random variables, under some conditions which are stronger than (2.1) and (2.2).

Theorem 2.3. Let $\left\{X_{n i}, 0 \leqslant i \leqslant l_{n}, n \geqslant 1\right\}$ be an array of rowwise pairwise NQD random variables and $1 \leqslant r<2$. Let $\left\{k_{n}, n \geqslant 1\right\}$ be a sequence of positive integers such that $k_{n} \uparrow \infty$ strictly and $h(n) / k_{n} \rightarrow 0$. Suppose the following conditions hold:

$$
\begin{gathered}
\sup _{n \geqslant 1} k_{n}^{-1} \sum_{i=0}^{l_{n}} \mathbf{E}\left|X_{n i}\right|^{r+\lambda}<\infty, \\
\lim _{n \rightarrow \infty} k_{n}^{-1} \sum_{i=0}^{l_{n}} \sup _{y \geqslant h(n)} y^{1+\eta} \mathbf{P}\left(\left|X_{n i}\right|^{r}>y\right)=0,
\end{gathered}
$$

where $0<\lambda<2-r$ and $\eta>2 / r-1$. Then

$$
\sum_{n=1}^{\infty} k_{n}^{-1} \mathbf{P}\left(\left|\sum_{i=0}^{l_{n}}\left(X_{n i}-\mathbf{E} X_{n i}\right)\right| \geqslant k_{n}^{1 / r} \varepsilon\right)<\infty \quad \forall \varepsilon>0 .
$$

3. Proofs. To prove our main results, we need some lemmas. Now we state the following lemma, which is similar to [14, Corollary 2.1].

Lemma 3.1. Let $\left\{X_{n i}, 0 \leqslant i \leqslant l_{n}, n \geqslant 1\right\}$ be an array of random variables and $r>0$. Let moreover $\{h(n), n \geqslant 1\}$ be an increasing sequence of positive constants with $h(n) \uparrow \infty$ as $n \uparrow \infty, k_{n} \rightarrow \infty$, and $h(n) / k_{n} \rightarrow 0$. Suppose (2.1) and (2.2) hold, then for all $\beta>r$

$$
\lim _{n \rightarrow \infty} k_{n}^{-\beta / r} \sum_{i=0}^{l_{n}} \mathbf{E}\left|X_{n i}\right|^{\beta} I\left(\left|X_{n i}\right|^{r} \leqslant k_{n}+1\right)=0 .
$$

$\mathrm{R}$ e $\mathrm{m}$ a $\mathrm{rk}$ 3.1. Under the same conditions as in Lemma 3.1, Wu and Guan [14] obtained

$$
\lim _{n \rightarrow \infty} k_{n}^{-\beta / r} \sum_{i=0}^{l_{n}} \mathbf{E}\left|X_{n i}\right|^{\beta} I\left(\left|X_{n i}\right|^{r} \leqslant k_{n}\right)=0 .
$$

Equality (3.1) follows from (3.2) immediately and hence we omit the proof.

The following lemma is well known (cf. [2]).

Lemma 3.2. Let $\left\{X_{n}, n \geqslant 1\right\}$ be a sequence of pairwise $N Q D$ random variables. Let $\left\{f_{n}, n \geqslant 1\right\}$ be a sequence of increasing functions. Then $\left\{f_{n}\left(X_{n}\right), n \geqslant 1\right\}$ is a sequence of pairwise $N Q D$ random variables.

In addition, we need the following lemmas (cf. [18, p. 101, 105]). 
Lemma 3.3. Let $0<p<\infty, X_{n} \in L^{p}$, and $X_{n} \rightarrow X$ in probability. Then the following three propositions are equivalent:

(i) $\left\{\left|X_{n}\right|^{p}\right\}$ is uniformly integrable;

(ii) $X_{n} \rightarrow X$ in $L^{p}$;

(iii) $\mathbf{E}\left|X_{n}\right|^{p} \rightarrow \mathbf{E}|X|^{p}<\infty$.

Lemma 3.4. If $\sup _{n \geqslant 1} \mathbf{E}\left|X_{n}\right|^{p}<\infty$ for some $p>1$, then $\left\{X_{n}\right\}$ is uniformly integrable.

P r o of of $\mathrm{Th}$ e ore $\mathrm{m}$ 2.1. Let

$$
\begin{aligned}
& Y_{n i}=-t^{1 / r} I\left(X_{n i}<-t^{1 / r}\right)+X_{n i} I\left(\left|X_{n i}\right| \leqslant t^{1 / r}\right)+t^{1 / r} I\left(X_{n i}>t^{1 / r}\right), \\
& Z_{n i}=X_{n i}-Y_{n i}=\left(X_{n i}+t^{1 / r}\right) I\left(X_{n i}<-t^{1 / r}\right)+\left(X_{n i}-t^{1 / r}\right) I\left(X_{n i}>t^{1 / r}\right) .
\end{aligned}
$$

By Lemma 3.2, we know that $\left\{Y_{n i}, 0 \leqslant i \leqslant l_{n}, n \geqslant 1\right\}$ and $\left\{Z_{n i}, 0 \leqslant i \leqslant l_{n}, n \geqslant 1\right\}$ are arrays of rowwise pairwise NQD. Given $\varepsilon>0$, we have

$$
\begin{aligned}
\mathbf{E}\left\{k_{n}^{-1 / r}\left|\sum_{i=0}^{l_{n}}\left(X_{n i}-\mathbf{E} X_{n i}\right)\right|\right\}^{r}= & k_{n}^{-1} \int_{0}^{\infty} \mathbf{P}\left(\left|\sum_{i=0}^{l_{n}}\left(X_{n i}-\mathbf{E} X_{n i}\right)\right| \geqslant t^{1 / r}\right) \mathrm{d} t \\
\leqslant & \varepsilon+k_{n}^{-1} \int_{k_{n} \varepsilon}^{\infty} \mathbf{P}\left(\left|\sum_{i=0}^{l_{n}}\left(X_{n i}-\mathbf{E} X_{n i}\right)\right| \geqslant t^{1 / r}\right) \mathrm{d} t \\
\leqslant & \varepsilon+k_{n}^{-1} \int_{k_{n} \varepsilon}^{\infty} \mathbf{P}\left(\left|\sum_{i=0}^{l_{n}}\left(Z_{n i}-\mathbf{E} Z_{n i}\right)\right| \geqslant \frac{t^{1 / r}}{2}\right) \mathrm{d} t \\
& +k_{n}^{-1} \int_{k_{n} \varepsilon}^{\infty} \mathbf{P}\left(\left|\sum_{i=0}^{l_{n}}\left(Y_{n i}-\mathbf{E} Y_{n i}\right)\right| \geqslant \frac{t^{1 / r}}{2}\right) \mathrm{d} t \\
= & : \varepsilon+I_{1}+I_{2} .
\end{aligned}
$$

To prove Theorem 2.1, it suffices to prove that $I_{1} \rightarrow 0$ and $I_{2} \rightarrow 0$ as $n \rightarrow \infty$.

Without loss of generality, we may assume $0<\varepsilon<1$. Since $h(n) / k_{n} \rightarrow 0$ as $n \rightarrow \infty$, there exists $N_{1}$ such that $h(n) \leqslant k_{n} \varepsilon$ if $n>N_{1}$.

Noting that $\left|Z_{n i}\right| \leqslant\left|X_{n i}\right| I\left(\left|X_{n i}\right|>t^{1 / r}\right)$, for all $t \geqslant k_{n} \varepsilon$, we have

$$
\begin{aligned}
\sup _{t \geqslant k_{n} \varepsilon}\left|t^{-1 / r} \sum_{i=0}^{l_{n}} \mathbf{E} Z_{n i}\right| & \leqslant \sup _{t \geqslant k_{n} \varepsilon} t^{-1 / r} \sum_{i=0}^{l_{n}} \mathbf{E}\left|Z_{n i}\right| \\
& \leqslant \sup _{t \geqslant k_{n} \varepsilon} t^{-1 / r} \sum_{i=0}^{l_{n}} \mathbf{E}\left|X_{n i}\right| I\left(\left|X_{n i}\right|>t^{1 / r}\right) \\
& \leqslant\left(k_{n} \varepsilon\right)^{-1 / r} \sum_{i=0}^{l_{n}} \mathbf{E}\left|X_{n i}\right| I\left(\left|X_{n i}\right|^{r}>k_{n} \varepsilon\right) \\
& \leqslant \varepsilon^{-1} k_{n}^{-1} \sum_{i=0}^{l_{n}} \mathbf{E}\left|X_{n i}\right|^{r} I\left(\left|X_{n i}\right|^{r}>k_{n} \varepsilon\right) \\
& \leqslant \varepsilon^{-1} k_{n}^{-1} \sum_{i=0}^{l_{n}} \mathbf{E}\left|X_{n i}\right|^{r} I\left(\left|X_{n i}\right|^{r}>h(n)\right) \rightarrow 0 \quad \text { as } n \rightarrow \infty .
\end{aligned}
$$

Therefore, there exists $N_{2}$ such that

$$
\left|\sum_{i=0}^{l_{n}} \mathbf{E} Z_{n i}\right| \leqslant \frac{t^{1 / r}}{4} \quad \text { if } n>N_{2}
$$


holds uniformly for $t \geqslant k_{n} \varepsilon$. Then, for $n>\max \left\{N_{1}, N_{2}\right\}$, we can get

$$
\begin{aligned}
I_{1} & =k_{n}^{-1} \int_{k_{n} \varepsilon}^{\infty} \mathbf{P}\left(\left|\sum_{i=0}^{l_{n}}\left(Z_{n i}-\mathbf{E} Z_{n i}\right)\right| \geqslant \frac{t^{1 / r}}{2}\right) \mathrm{d} t \\
& \leqslant k_{n}^{-1} \int_{k_{n} \varepsilon}^{\infty} \mathbf{P}\left(\left|\sum_{i=0}^{l_{n}} Z_{n i}\right|+\left|\sum_{i=0}^{l_{n}} \mathbf{E} Z_{n i}\right| \geqslant \frac{t^{1 / r}}{2}\right) \mathrm{d} t \\
& \leqslant k_{n}^{-1} \int_{k_{n} \varepsilon}^{\infty} \mathbf{P}\left(\left|\sum_{i=0}^{l_{n}} Z_{n i}\right| \geqslant \frac{t^{1 / r}}{4}\right) \mathrm{d} t \\
& \leqslant k_{n}^{-1} \int_{k_{n} \varepsilon}^{\infty} \mathbf{P}\left(\exists i: 0 \leqslant i \leqslant l_{n} \text { such that }\left|X_{n i}\right|>t^{1 / r}\right) \mathrm{d} t \\
& \leqslant k_{n}^{-1} \int_{k_{n} \varepsilon}^{\infty} \sum_{i=0}^{l_{n}} \mathbf{P}\left(\left|X_{n i}\right|>t^{1 / r}\right) \mathrm{d} t \leqslant k_{n}^{-1} \sum_{i=0}^{l_{n}} \mathbf{E}\left|X_{n i}\right|^{r} I\left(\left|X_{n i}\right|^{r}>k_{n} \varepsilon\right) \\
& \leqslant k_{n}^{-1} \sum_{i=0}^{l_{n}} \mathbf{E}\left|X_{n i}\right|^{r} I\left(\left|X_{n i}\right|^{r}>h(n)\right) \rightarrow 0 \text { as } n \rightarrow \infty .
\end{aligned}
$$

Then we prove that $I_{2} \rightarrow 0$ as $n \rightarrow \infty$. By the Markov inequality, we have

$$
\begin{aligned}
I_{2} & \leqslant C k_{n}^{-1} \int_{k_{n} \varepsilon}^{\infty} t^{-2 / r} \mathbf{E}\left(\sum_{i=0}^{l_{n}}\left(Y_{n i}-\mathbf{E} Y_{n i}\right)\right)^{2} \mathrm{~d} t \\
& =C k_{n}^{-1} \int_{k_{n} \varepsilon}^{\infty} t^{-2 / r}\left(\sum_{i=0}^{l_{n}} \mathbf{E}\left(Y_{n i}-\mathbf{E} Y_{n i}\right)^{2}+\sum_{i \neq j} \operatorname{Cov}\left(Y_{n i}, Y_{n j}\right)\right) \mathrm{d} t \\
& \leqslant C k_{n}^{-1} \int_{k_{n} \varepsilon}^{\infty} t^{-2 / r} \sum_{i=0}^{l_{n}} \mathbf{E}\left(Y_{n i}-\mathbf{E} Y_{n i}\right)^{2} \mathrm{~d} t \leqslant C k_{n}^{-1} \int_{k_{n} \varepsilon}^{\infty} t^{-2 / r} \sum_{i=0}^{l_{n}} \mathbf{E} Y_{n i}^{2} \mathrm{~d} t \\
& =C k_{n}^{-1} \int_{k_{n} \varepsilon}^{\infty} t^{-2 / r} \sum_{i=0}^{l_{n}} \mathbf{E} X_{n i}^{2} I\left(\left|X_{n i}\right|^{r} \leqslant t\right) \mathrm{d} t+C k_{n}^{-1} \int_{k_{n} \varepsilon}^{\infty} \sum_{i=0}^{l_{n}} \mathbf{P}\left(\left|X_{n i}\right|>t^{1 / r}\right) \mathrm{d} t \\
& =: I_{3}+I_{4} .
\end{aligned}
$$

Following the proof of $I_{1} \rightarrow 0$, we have $I_{4} \rightarrow 0$. Next we prove $I_{3} \rightarrow 0$. Let $d_{n}=$ $\left[k_{n}\right]+1$, then

$$
\begin{aligned}
I_{3}= & C k_{n}^{-1} \int_{k_{n} \varepsilon}^{\infty} t^{-2 / r} \sum_{i=0}^{l_{n}} \mathbf{E} X_{n i}^{2} I\left(\left|X_{n i}\right|^{r} \leqslant d_{n} \varepsilon\right) \mathrm{d} t \\
& +C k_{n}^{-1} \int_{k_{n} \varepsilon}^{\infty} t^{-2 / r} \sum_{i=0}^{l_{n}} \mathbf{E} X_{n i}^{2} I\left(d_{n} \varepsilon<\left|X_{n i}\right|^{r} \leqslant t\right) \mathrm{d} t \\
= & C k_{n}^{-1} \sum_{i=0}^{l_{n}} \mathbf{E} X_{n i}^{2} I\left(\left|X_{n i}\right|^{r} \leqslant d_{n} \varepsilon\right) \int_{k_{n} \varepsilon}^{\infty} t^{-2 / r} \mathrm{~d} t \\
& +C k_{n}^{-1} \int_{d_{n} \varepsilon}^{\infty} t^{-2 / r} \sum_{i=0}^{l_{n}} \mathbf{E} X_{n i}^{2} I\left(d_{n} \varepsilon<\left|X_{n i}\right|^{r} \leqslant t\right) \mathrm{d} t \\
= & I_{31}+I_{32} .
\end{aligned}
$$

By [14, Remark 2.1], we know that the conditions of $h$-integrability with exponent $r$ imply (2.1) and (2.2). Take $\beta=2$ in (3.1), then by Lemma 3.1 and $r<2$, we have

$$
I_{31} \leqslant C \varepsilon^{-2 / r+1} k_{n}^{-2 / r} \sum_{i=0}^{l_{n}} \mathbf{E} X_{n i}^{2} I\left(\left|X_{n i}\right|^{r} \leqslant d_{n} \varepsilon\right) \quad(\text { since } 0<\varepsilon<1)
$$




$$
\leqslant C \varepsilon^{-2 / r+1} k_{n}^{-2 / r} \sum_{i=0}^{l_{n}} \mathbf{E} X_{n i}^{2} I\left(\left|X_{n i}\right|^{r} \leqslant k_{n}+1\right) \rightarrow 0 \quad \text { as } n \rightarrow \infty .
$$

For $I_{32}$, we have

$$
\begin{aligned}
I_{32} & =C k_{n}^{-1} \sum_{i=0}^{l_{n}} \sum_{m=d_{n}}^{\infty} \int_{m \varepsilon}^{(m+1) \varepsilon} t^{-2 / r} \mathbf{E} X_{n i}^{2} I\left(d_{n} \varepsilon<\left|X_{n i}\right|^{r} \leqslant t\right) \mathrm{d} t \\
& \leqslant C k_{n}^{-1} \sum_{i=0}^{l_{n}} \sum_{m=d_{n}}^{\infty} m^{-2 / r} \mathbf{E} X_{n i}^{2} I\left(d_{n} \varepsilon<\left|X_{n i}\right|^{r} \leqslant(m+1) \varepsilon\right) \\
& \leqslant C k_{n}^{-1} \sum_{i=0}^{l_{n}} \sum_{m=d_{n}}^{\infty} m^{-2 / r} \sum_{s=d_{n}}^{m} \mathbf{E} X_{n i}^{2} I\left(s \varepsilon<\left|X_{n i}\right|^{r} \leqslant(s+1) \varepsilon\right) \\
& \leqslant C k_{n}^{-1} \sum_{i=0}^{l_{n}} \sum_{s=d_{n}}^{\infty} \mathbf{E} X_{n i}^{2} I\left(s \varepsilon<\left|X_{n i}\right|^{r} \leqslant(s+1) \varepsilon\right) \sum_{m=s}^{\infty} m^{-2 / r} \\
& \leqslant C k_{n}^{-1} \sum_{i=0}^{l_{n}} \sum_{s=d_{n}}^{\infty} s^{1-2 / r} \mathbf{E} X_{n i}^{2} I\left(s \varepsilon<\left|X_{n i}\right|^{r} \leqslant(s+1) \varepsilon\right) \\
& \leqslant C k_{n}^{-1} \sum_{i=0}^{l_{n}} \mathbf{E}\left|X_{n i}\right|^{r} I\left(\left|X_{n i}\right|^{r}>d_{n} \varepsilon\right) \quad\left(\text { since } d_{n}>k_{n}\right) \\
& \leqslant C k_{n}^{-1} \sum_{i=0}^{l_{n}} \mathbf{E}\left|X_{n i}\right|^{r} I\left(\left|X_{n i}\right|^{r}>h(n)\right) \rightarrow 0 \quad \text { as } n \rightarrow \infty .
\end{aligned}
$$

The proof is complete.

P r o of of $\mathrm{T}$ h e o r e m 2.2. Let $S_{n}=\sum_{i=0}^{l_{n}}\left(X_{n i}-\mathbf{E} X_{n i}\right)$ and

$$
\begin{aligned}
& Y_{n i}^{\prime}=-k_{n}^{1 / r} I\left(X_{n i}<-k_{n}^{1 / r}\right)+X_{n i} I\left(\left|X_{n i}\right| \leqslant k_{n}^{1 / r}\right)+k_{n}^{1 / r} I\left(X_{n i}>k_{n}^{1 / r}\right), \\
& Z_{n i}^{\prime}=X_{n i}-Y_{n i}^{\prime}=\left(X_{n i}+k_{n}^{1 / r}\right) I\left(X_{n i}<-k_{n}^{1 / r}\right)+\left(X_{n i}-k_{n}^{1 / r}\right) I\left(X_{n i}>k_{n}^{1 / r}\right) .
\end{aligned}
$$

By Lemma 3.3, to prove Theorem 2.2, it suffices to show that $k_{n}^{-1 / r} S_{n} \rightarrow 0$ in probability and $\left\{\left|k_{n}^{-1 / r} S_{n}\right|^{p}, n \geqslant 1\right\}$ is uniformly integrable.

First we prove that $k_{n}^{-1 / r} S_{n} \rightarrow 0$ in probability. We can get

$$
\begin{aligned}
\mathbf{P}\left(k_{n}^{-1 / r}\left|S_{n}\right| \geqslant \varepsilon\right) \leqslant & \mathbf{P}\left(\left|\sum_{i=0}^{l_{n}}\left(Y_{n i}^{\prime}-\mathbf{E} Y_{n i}^{\prime}\right)\right| \geqslant k_{n}^{1 / r} \varepsilon / 2\right) \\
& +\mathbf{P}\left(\left|\sum_{i=0}^{l_{n}}\left(Z_{n i}^{\prime}-\mathbf{E} Z_{n i}^{\prime}\right)\right| \geqslant \frac{k_{n}^{1 / r} \varepsilon}{2}\right) \\
=: & I_{5}+I_{6} .
\end{aligned}
$$

By the Markov inequality and (1.1), we have

$$
\begin{aligned}
I_{5} & \leqslant C k_{n}^{-2 / r} \sum_{i=0}^{l_{n}} \mathbf{E}\left(Y_{n i}^{\prime}-\mathbf{E} Y_{n i}^{\prime}\right)^{2} \leqslant C k_{n}^{-2 / r} \sum_{i=0}^{l_{n}} \mathbf{E}\left(Y_{n i}^{\prime}\right)^{2} \\
& =C k_{n}^{-2 / r} \sum_{i=0}^{l_{n}} \mathbf{E} X_{n i}^{2} I\left(\left|X_{n i}\right|^{r} \leqslant k_{n}\right)+C \sum_{i=0}^{l_{n}} \mathbf{P}\left(\left|X_{n i}\right|^{r}>k_{n}\right) \\
& =: I_{51}+I_{52} .
\end{aligned}
$$

Take $\beta=2$ in (3.1), then by Lemma 3.1, we have $I_{51} \rightarrow 0$ as $n \rightarrow \infty$. Taking $y=k_{n}$ in (2.2), we have $I_{52} \rightarrow 0$ as $n \rightarrow \infty$. 
For $I_{6}$, noting that $\left|Z_{n i}^{\prime}\right| \leqslant\left|X_{n i}\right| I\left(\left|X_{n i}\right|^{r}>k_{n}\right)$, by the Markov inequality and $C_{r^{-}}$ inequality, we have

$$
\begin{aligned}
I_{6} \leqslant & C k_{n}^{-1 / r} \mathbf{E}\left|\sum_{i=0}^{l_{n}}\left(Z_{n i}^{\prime}-Z_{n i}^{\prime}\right)\right| \leqslant C k_{n}^{-1 / r} \sum_{i=0}^{l_{n}} \mathbf{E}\left|Z_{n i}^{\prime}\right| \\
\leqslant & C k_{n}^{-1 / r} \sum_{i=0}^{l_{n}} \mathbf{E}\left|X_{n i}\right| I\left(\left|X_{n i}\right|^{r}>k_{n}\right) \\
= & C k_{n}^{-1 / r} \sum_{i=0}^{l_{n}} \int_{0}^{k_{n}^{1 / r}} \mathbf{P}\left(\left|X_{n i}\right| I\left(\left|X_{n i}\right|^{r}>k_{n}\right)>t\right) \mathrm{d} t \\
& +C k_{n}^{-1 / r} \sum_{i=0}^{l_{n}} \int_{k_{n}^{1 / r}}^{\infty} \mathbf{P}\left(\left|X_{n i}\right| I\left(\left|X_{n i}\right|^{r}>k_{n}\right)>t\right) \mathrm{d} t \\
& \leqslant C \sum_{i=0}^{l_{n}} \mathbf{P}\left(\left|X_{n i}\right|^{r}>k_{n}\right)+C k_{n}^{-1 / r} \sum_{i=0}^{l_{n}} \int_{k_{n}^{1 / r}}^{\infty} \mathbf{P}\left(\left|X_{n i}\right|>t\right) \mathrm{d} t \\
= & I_{61}+I_{62} .
\end{aligned}
$$

By an argument similar to the proof that $I_{52} \rightarrow 0$, we have $I_{61} \rightarrow 0$. Then we consider $I_{62}$. From (2.2), we know that, given $\varepsilon>0$, there exists $N_{3}$ such that $k_{n}^{-1} \sum_{i=0}^{l_{n}} y \mathbf{P}\left(\left|X_{n i}\right|^{r} \geqslant\right.$ $y) \leqslant \varepsilon$ if $n>N_{3}$. Let $t=y^{1 / r}$, then for $n>N_{3}$, we have

$$
\begin{aligned}
I_{62} & =C k_{n}^{-1 / r} \sum_{i=0}^{l_{n}} \int_{k_{n}}^{\infty} y^{1 / r-1} \mathbf{P}\left(\left|X_{n i}\right|^{r}>y\right) \mathrm{d} y \\
& \leqslant C \varepsilon k_{n}^{1-1 / r} \int_{k_{n}}^{\infty} y^{1 / r-2} \mathrm{~d} y \leqslant C \varepsilon \frac{r}{r-1} .
\end{aligned}
$$

Since $\varepsilon>0$ is arbitrary, $I_{62} \rightarrow 0$ as $n \rightarrow \infty$. The proof of $k_{n}^{-1 / r} S_{n} \rightarrow 0$ in probability is complete.

Next we prove that $\left\{\left|k_{n}^{-1 / r} S_{n}\right|^{p}, n \geqslant 1\right\}$ is uniformly integrable. Let $Y_{n i}$ and $Z_{n i}$ be the same as in the proof of Theorem 2.1. Since $r / p>1$, by Lemma 3.4 we know that it suffices to show $\sup _{n \geqslant 1} \mathbf{E}\left(\left|k_{n}^{-1 / r} S_{n}\right|^{p}\right)^{r / p}<\infty$. We have

$$
\begin{aligned}
\sup _{n \geqslant 1} \mathbf{E}\left(\left|k_{n}^{-1 / r} S_{n}\right|^{p}\right)^{r / p}= & \sup _{n \geqslant 1} k_{n}^{-1} \int_{0}^{\infty} \mathbf{P}\left(\left|S_{n}\right| \geqslant t^{1 / r}\right) \mathrm{d} t \\
\leqslant & 2+\sup _{n \geqslant 1} k_{n}^{-1} \int_{\left[k_{n}\right]+1}^{\infty} \mathbf{P}\left(\left|S_{n}\right| \geqslant t^{1 / r}\right) \mathrm{d} t \\
\leqslant & 2+\sup _{n \geqslant 1} k_{n}^{-1} \int_{\left[k_{n}\right]+1}^{\infty} \mathbf{P}\left(\left|\sum_{i=0}^{l_{n}}\left(Z_{n i}-\mathbf{E} Z_{n i}\right)\right| \geqslant \frac{t^{1 / r}}{2}\right) \mathrm{d} t \\
& +\sup _{n \geqslant 1} k_{n}^{-1} \int_{\left[k_{n}\right]+1}^{\infty} \mathbf{P}\left(\left|\sum_{i=0}^{l_{n}}\left(Y_{n i}-\mathbf{E} Y_{n i}\right)\right| \geqslant \frac{t^{1 / r}}{2}\right) \mathrm{d} t \\
= & : 2+I_{7}+I_{8} .
\end{aligned}
$$

For $I_{7}$, by following the proof of $I_{6} \rightarrow 0$, we have

$$
\begin{aligned}
\sup _{t \geqslant\left[k_{n}\right]+1} t^{-1 / r}\left|\sum_{i=0}^{l_{n}} \mathbf{E} Z_{n i}\right| & \leqslant \sup _{t \geqslant\left[k_{n}\right]+1} t^{-1 / r} \sum_{i=0}^{l_{n}} \mathbf{E}\left|Z_{n i}\right| \\
& \leqslant \sup _{t \geqslant\left[k_{n}\right]+1} t^{-1 / r} \sum_{i=0}^{l_{n}} \mathbf{E}\left|X_{n i}\right| I\left(\left|X_{n i}\right|^{r}>t\right)
\end{aligned}
$$




$$
\begin{aligned}
& \leqslant\left(\left[k_{n}\right]+1\right)^{-1 / r} \sum_{i=0}^{l_{n}} \mathbf{E}\left|X_{n i}\right| I\left(\left|X_{n i}\right|^{r}>\left[k_{n}\right]+1\right) \\
& \leqslant k_{n}^{-1 / r} \sum_{i=0}^{l_{n}} \mathbf{E}\left|X_{n i}\right| I\left(\left|X_{n i}\right|^{r}>k_{n}\right) \rightarrow 0 \quad \text { as } n \rightarrow \infty .
\end{aligned}
$$

Therefore, by an argument similar to the proof that $I_{1} \rightarrow 0$, we have

$$
I_{7} \leqslant \sup _{n \geqslant 1} k_{n}^{-1} \sum_{i=0}^{l_{n}} \int_{0}^{\infty} \mathbf{P}\left(\left|X_{n i}\right|>t^{1 / r}\right) \mathrm{d} t=\sup _{n \geqslant 1} k_{n}^{-1} \sum_{i=0}^{l_{n}} \mathbf{E}\left|X_{n i}\right|^{r}<\infty .
$$

For $I_{8}$, by following the proof of $I_{2} \rightarrow 0$, we have

$$
\begin{aligned}
I_{8} \leqslant & C \sup _{n \geqslant 1} k_{n}^{-1} \sum_{i=0}^{l_{n}} \int_{\left[k_{n}\right]+1}^{\infty} t^{-2 / r} \mathbf{E} X_{n i}^{2} I\left(\left|X_{n i}\right|^{r} \leqslant t\right) \mathrm{d} t \\
& +C \sup _{n \geqslant 1} k_{n}^{-1} \sum_{i=0}^{l_{n}} \int_{\left[k_{n}\right]+1}^{\infty} \mathbf{P}\left(\left|X_{n i}\right|>t^{1 / r}\right) \mathrm{d} t \\
=: & I_{8}^{\prime}+I_{8}^{\prime \prime} .
\end{aligned}
$$

By an argument similar to the proof that $I_{7}<\infty$, we have $I_{8}^{\prime \prime}<\infty$. For $I_{8}^{\prime}$, by an argument similar to the proof that $I_{32} \rightarrow 0$, we have

$$
\begin{aligned}
I_{8}^{\prime} \leqslant & C \sup _{n \geqslant 1} k_{n}^{-1} \sum_{i=0}^{l_{n}} \sum_{m=\left[k_{n}\right]+1}^{\infty} m^{-2 / r} \mathbf{E} X_{n i}^{2} I\left(\left|X_{n i}\right|^{r} \leqslant m+1\right) \\
\leqslant & C \sup _{n \geqslant 1} k_{n}^{-1} \sum_{i=0}^{l_{n}} \sum_{m=1}^{\infty} m^{-2 / r} \mathbf{E} X_{n i}^{2} I\left(\left|X_{n i}\right|^{r} \leqslant m+1\right) \\
\leqslant & C \sup _{n \geqslant 1} k_{n}^{-1} \sum_{i=0}^{l_{n}} \sum_{m=1}^{\infty} m^{-2 / r} \mathbf{E} X_{n i}^{2} I\left(\left|X_{n i}\right|^{r} \leqslant 1\right) \quad(\text { since } r<2) \\
& +C \sup _{n \geqslant 1} k_{n}^{-1} \sum_{i=0}^{l_{n}} \sum_{m=1}^{\infty} m^{-2 / r} \sum_{s=1}^{m} \mathbf{E} X_{n i}^{2} I\left(s<\left|X_{n i}\right|^{r} \leqslant s+1\right) \\
\leqslant & C \sup _{n \geqslant 1} k_{n}^{-1} \sum_{i=0}^{l_{n}} \mathbf{E}\left|X_{n i}\right|^{r} I\left(\left|X_{n i}\right|^{r} \leqslant 1\right) \\
& +C \sup _{n \geqslant 1} k_{n}^{-1} \sum_{i=0}^{l_{n}} \sum_{s=1}^{\infty} \mathbf{E} X_{n i}^{2} I\left(s<\left|X_{n i}\right|^{r} \leqslant(s+1)\right) \sum_{m=s}^{\infty} m^{-2 / r} \\
\leqslant & C+C \sup _{n \geqslant 1} k_{n}^{-1} \sum_{i=0}^{l_{n}} \sum_{s=1}^{\infty} s^{1-2 / r} \mathbf{E} X_{n i}^{2} I\left(s<\left|X_{n i}\right|^{r} \leqslant s+1\right) \\
\leqslant & C+C \sup _{n \geqslant 1} k_{n}^{-1} \sum_{i=0}^{l_{n}} \mathbf{E}\left|X_{n i}\right|^{r} I\left(\left|X_{n i}\right|^{r}>1\right)<\infty .
\end{aligned}
$$

The proof is complete.

P r o of of $\mathrm{T}$ h e o r e $\mathrm{m}$ 2.3. Let $x=h(n)$ and

$$
\begin{aligned}
& Y_{n i}=-x^{1 / r} I\left(X_{n i}<-x^{1 / r}\right)+X_{n i} I\left(\left|X_{n i}\right| \leqslant x^{1 / r}\right)+x^{1 / r} I\left(X_{n i}>x^{1 / r}\right), \\
& Z_{n i}=X_{n i}-Y_{n i}=\left(X_{n i}+x^{1 / r}\right) I\left(X_{n i}<-x^{1 / r}\right)+\left(X_{n i}-x^{1 / r}\right) I\left(X_{n i}>x^{1 / r}\right) .
\end{aligned}
$$

For given $\varepsilon>0$, we can get

$$
\sum_{n=1}^{\infty} k_{n}^{-1} \mathbf{P}\left(\left|\sum_{i=0}^{l_{n}}\left(X_{n i}-\mathbf{E} X_{n i}\right)\right| \geqslant k_{n}^{1 / r} \varepsilon\right)
$$




$$
\begin{aligned}
\leqslant & \sum_{n=1}^{\infty} k_{n}^{-1} \mathbf{P}\left(\left|\sum_{i=0}^{l_{n}}\left(Y_{n i}-\mathbf{E} Y_{n i}\right)\right| \geqslant \frac{k_{n}^{1 / r} \varepsilon}{2}\right) \\
& +\sum_{n=1}^{\infty} k_{n}^{-1} \mathbf{P}\left(\left|\sum_{i=0}^{l_{n}}\left(Z_{n i}-\mathbf{E} Z_{n i}\right)\right| \geqslant \frac{k_{n}^{1 / r} \varepsilon}{2}\right) \\
= & : I_{9}+I_{10} .
\end{aligned}
$$

For $I_{9}$, by the Markov inequality and $C_{r}$-inequality, we have

$$
\begin{aligned}
I_{9} \leqslant & C \sum_{n=1}^{\infty} k_{n}^{-1-2 / r} \sum_{i=0}^{l_{n}} \mathbf{E}\left(Y_{n i}-\mathbf{E} Y_{n i}\right)^{2} \\
\leqslant & C \sum_{n=1}^{\infty} k_{n}^{-1-2 / r} \sum_{i=0}^{l_{n}} \mathbf{E} X_{n i}^{2} I\left(\left|X_{n i}\right|^{r} \leqslant h(n)\right) \\
& +C \sum_{n=1}^{\infty} k_{n}^{-1-2 / r}(h(n))^{2 / r} \sum_{i=0}^{l_{n}} \mathbf{P}\left(\left|X_{n i}\right|^{r}>h(n)\right) \\
= & I_{9}^{\prime}+I_{9}^{\prime \prime} .
\end{aligned}
$$

From (2.4), we know that there exists $N$ such that

$$
\sup _{y \geqslant h(n)} k_{n}^{-1} \sum_{i=0}^{l_{n}} \mathbf{P}\left(\left|X_{n i}\right|^{r}>y\right) \leqslant \sup _{y \geqslant h(n)} y^{-1-\eta}=(h(n))^{-1-\eta} \quad \text { if } n>N .
$$

Then for $n>N$, since $r<2$ and $2 / r-1-\eta<0$, we have

$$
\begin{aligned}
I_{9}^{\prime \prime} & \leqslant C+C \sum_{n=N+1}^{\infty} k_{n}^{-1-2 / r}(h(n))^{2 / r} \sum_{i=0}^{l_{n}} \mathbf{P}\left(\left|X_{n i}\right|^{r}>h(n)\right) \\
& \leqslant C+C \sum_{n=N+1}^{\infty} k_{n}^{-2 / r}(h(n))^{2 / r-1-\eta}<\infty .
\end{aligned}
$$

For $I_{9}^{\prime}$, using $(r+\lambda) / 2<1,(2.3)$, and $h(n) / k_{n} \rightarrow 0$, we have

$$
\begin{aligned}
I_{9}^{\prime} & =C \sum_{n=1}^{\infty} k_{n}^{-1-2 / r}(h(n))^{2 / r} \sum_{i=0}^{l_{n}} \mathbf{E}\left[\frac{X_{n i}}{(h(n))^{1 / r}}\right]^{2} I\left(\left|X_{n i}\right|^{r} \leqslant h(n)\right) \\
& \leqslant C \sum_{n=1}^{\infty} k_{n}^{-1-2 / r}(h(n))^{2 / r-1-\lambda / r} \sum_{i=0}^{l_{n}} \mathbf{E}\left|X_{n i}\right|^{r+\lambda} I\left(\left|X_{n i}\right|^{r} \leqslant h(n)\right) \\
& \leqslant C\left(\sup _{n \geqslant 1} k_{n}^{-1} \sum_{i=0}^{l_{n}} \mathbf{E}\left|X_{n i}\right|^{r+\lambda}\right) \sum_{n=1}^{\infty} k_{n}^{-1-\lambda / r}\left[\frac{h(n)}{k_{n}}\right]^{2 / r-1-\lambda / r}<\infty .
\end{aligned}
$$

For $I_{10}$, by an argument similar to the proof that $I_{6} \rightarrow 0$, the Markov inequality and $C_{r}$-inequality, we have

$$
\begin{aligned}
I_{10} & \leqslant C \sum_{n=1}^{\infty} k_{n}^{-1-2 / r} \sum_{i=0}^{l_{n}} \mathbf{E}\left(Z_{n i}-\mathbf{E} Z_{n i}\right)^{2} \\
& \leqslant C \sum_{n=1}^{\infty} k_{n}^{-1-2 / r} \sum_{i=0}^{l_{n}} \mathbf{E} X_{n i}^{2} I\left(\left|X_{n i}\right|^{r}>h(n)\right) \\
& \leqslant C \sum_{n=1}^{\infty} k_{n}^{-1-2 / r} \sum_{i=0}^{l_{n}}(h(n))^{2 / r} \mathbf{P}\left(\left|X_{n i}\right|^{r}>h(n)\right)
\end{aligned}
$$




$$
\begin{aligned}
& +C \sum_{n=1}^{\infty} k_{n}^{-1-2 / r} \sum_{i=0}^{l_{n}} \int_{(h(n))^{2 / r}}^{\infty} \mathbf{P}\left(X_{n i}^{2}>t\right) \mathrm{d} t \\
= & : I_{10}^{\prime}+I_{10}^{\prime \prime} .
\end{aligned}
$$

By an argument similar to the proof that $I_{9}^{\prime \prime}<\infty$, we can get $I_{10}^{\prime}<\infty$. Then we prove $I_{10}^{\prime \prime}<\infty$. Let $t=y^{2 / r} ;$ using (3.3), we have

$$
\begin{aligned}
I_{10}^{\prime \prime} & \leqslant C \sum_{n=1}^{\infty} k_{n}^{-1-2 / r} \sum_{i=0}^{l_{n}} \int_{h(n)}^{\infty} y^{2 / r-1} \mathbf{P}\left(\left|X_{n i}\right|^{r}>y\right) \mathrm{d} y \\
& \leqslant C+C \sum_{n=N+1}^{\infty} k_{n}^{-2 / r} \int_{h(n)}^{\infty} y^{2 / r-2-\eta} \mathrm{d} y \\
& \leqslant C+C \sum_{n=N+1}^{\infty} k_{n}^{-2 / r}(h(n))^{2 / r-1-\eta}<\infty
\end{aligned}
$$

The proof is complete.

Acknowledgments. The author is grateful to the referee for carefully reading the manuscript and for providing some comments and suggestions which led to improvements in the paper.

\section{REFERENCES}

1. Sung H.S., Lisawadi S., Volodin A. Weak laws of large numbers for arrays under a condition of uniform integrability. - J. Korean Math. Soc., 2008, v. 45, p. 289-300.

2. Lehmann E. L. Some concepts of dependence. - Ann. Math. Statist., 1966, v. 37, p. 1137-1153.

3. Joag-Dev K., Proschan F. Negative association of random variables, with applications. - Ann. Statist., 1983, v. 11, № 1, p. 286-295.

4. Newman C.M. Asymptotic independence and limit theorems for positively and negatively dependent random variables. - Statistics and Probability, v. 5. Ed. by Y. L. Tong. Hayward: Inst. Math. Statist., 1984, p. 127-140.

5. Wu Q. Y. Convergence properties of pairwise NQD random sequences. - Acta Math. Sin. (Engl. Ser.), 2002, v. 45, № 3, p. 617-624 (in Chinese).

6. Matula $P$. A note on the almost sure convergence of sums of negatively dependent random variables. - Statist. Probab. Lett., 1992, v. 15, № 3, p. 209-213.

7. Liang H. Y., Chen Z. J., Su C. Convergence of Jamison-type weighted sums of pairwise negatively quadrant dependent random variables. - Acta Math. Appl. Sin. (Engl. Ser.), 2002, v. 18, № 1, p. 161-168.

8. Li R., Yang W. G. Strong convergence of pairwise NQD random sequences. - J. Math. Anal. Appl., 2008, v. 344, № 2, p. 741-747.

9. Wan C. G. Law of large numbers and complete convergence for pairwise NQD random sequences. - Acta Math. Appl. Sin., 2005, v. 28, p. 253-261 (in Chinese).

10. Meng Y. J., Lin Z. Y. On the weak laws of large numbers for arrays of random variables. - Statist. Probab. Lett., 2009, v. 79, № 23, p. 2405-2414.

11. Gan S.X., Chen P. Y. Some limit theorems for sequences of pairwise NQD random variables. - Acta Math. Sci., Ser. B, Engl. Ed., 2008, v. 28, № 2, p. 269-281.

12. Baek J.I., Ko M. H., Kim T.S. On the complete convergence for weighted sums of dependent random variables under condition of weighted integrability. - J. Korean Math. Soc., 2008, v. 45, № 4, p. 1101-1111.

13. Ordóñez Cabrera M., Volodin A. Mean convergence theorems and weak laws of large numbers for weighted sums of random variables under a condition of weighted integrability. - J. Math. Anal. Appl., 2005, v. 305, № 2, p. 644-658. 
14. Wu Y.F., Guan M. Mean convergence theorems and weak laws of large numbers for weighted sums of dependent random variables. - J. Math. Anal. Appl., 2011, v. 377, № 2, p. 613-623.

15. Chen P. Y., Ordóñez Cabrera M., Volodin A. $L_{1}$-convergence for weighted sums of some dependent random variables. - Stoch. Anal. Appl., 2010, v. 28, №6, p. 928936.

16. Adler A., Rosalsky A., Volodin A. A mean convergence theorem and weak law for arrays of random elements in martingale type $p$ Banach spaces. - Statist. Probab. Lett., 1997, v. 32, № 2, p. 167-174.

17. Yang S. C. Almost sure convergence of weighted sums of mixing sequences. J. Systems Sci. Math. Sci., 1995, v. 15, № 3, p. 254-265 (in Chinese).

18. Chung K. L. A Course in Probability Theory. Orlando: Academic Press, 2001, 419 p.

Поступила в редакцию

3.X.2011

Исправленный вариант

21.XI.2013 